\title{
Survey on Mites Associated with Major Insect Pests Infesting Stored Grains in Middle Delta
}

\author{
Ferial M. A. El-Sayed ${ }^{1}$ and Mona M. A. Ghallab ${ }^{2}$ \\ ${ }^{1}$ Stored Grain Insects Department, Plant Protection Research Institute. ARC. \\ ${ }^{2}$ Acarology Department, Plant Protection Research Institute. ARC.
}

\begin{abstract}
The present study was directed towards the habitat of various mite species as predators, fungivores or as parasites associated with insects inhabiting grains and stored products. Samples were collected monthly from maize, wheat flour, broad bean, wheat and rough rice in four governorates, Kafr El-Sheikh, Dakahleya, Gharbeya and Menoufeya during the period between March 2003 and February 2004. The results revealed that: 1) The mite survey revealed the occurrence of 13 predatory species belonging to one family of the suborder Gamasida, and 6 families of the suborder Actinedida. Also 7 species belong to 4 families of the suborder Acaridida, were recorded as scavengers or fungivores. 2) The most abundant predaceous mites were: Blattisocius keegani Fox followed by Cheyletus malaccensis Oudemans, then Blattisocius tarsalis (Berlese). 3) The most abundant fungivore mites were: Tyrophagus putrescentiae (Shranck), Rhizoglyphus robini Claparède, and Acarus siro Linnaeus. 4) The most infested stored grains with mites was maize followed by wheat, rough rice, broad bean seeds then wheat flour. 5) The major insect pests of stored grains and seeds and associated mites were listed herein.
\end{abstract}

KEY WORDS: Mites, fungivores, predators, stored grains.

\section{INTRODUCTION}

Stored grains provide all of the essential nutritive requirements for insects and mites capable of chewing into the hard kernels. The biotic potential of these species is enormous and their activities cause heating of grain mass and moisture translocation which permit the development of molds and germination of the grain, and the pests proliferate.

Large numbers of mites are known to infest a variety of grains and stored products throughout the world. They attack damaged and undamaged grains devouring the embryos and other surrounding tissues. This prevents grain germination and reduces its nutritive value. They also feed on fungi and other micro-organisms. Contamination by alive and dead mite different stages as well as exuviae and feaces results in being harmful for human consumption. (Hughes 1976). Another group of mites acts as predators or parasites that prey on the eggs and developmental stages of the major and minor pests of the stored grains (Barker 1967).

Studies on mites inhabiting grains and stored products were reported previously by (Hegazy, 1961; Wafa et al., 1966; Attiah, 1969; Hughes, 1976; Taha, 1985; Zaher et al., 1985) and recently, by (Hoda et al., 1990; Fawzy, 1996; Halawa, 2003 and El-Sanady 2005). The present work was conducted to survey mites associated with stored grains in four governorates in Nile Delta.

\section{MATERIALS AND METHODS}

During the period between March 2003 and February 2004, samples of maize, wheat, wheat flour, broad bean and rough rice $^{1}$ were collected monthly from granaries at Kafr El-Sheikh, Dakahleya, Gharbeya and Menoufeya. Samples about $1 \mathrm{~kg}$. each, from the previous materials were collected in cloth bags and transported to the laboratory with a label concerning habitat locality and date of collection.

For extracting mites, an amount of $150 \mathrm{gm}$. from each sample under study was placed in modified Tullgren funnels and left for a period of 24 hours. The extracted mites were received in Petri-dishes filled with water and its edges smeared with a layer of Vaseline to prevent mites from escaping.

Extracted mites were cleared in Nesbitt's solution, then mounted in Hoyer's medium on glass slides. Specimens were identified and classified into their taxonomical rank by using different specific keys.

\section{RESULTS AND DISCUSSION}

\section{Mite diversity and species composition of stored products:}

Survey study reveals the occurrence of mites and insects inhabiting different stored crops (maize, wheat, broad bean, rough rice and wheat flour).

They feed on stored materials, fungus or being predators or parasites and the latters may be of great benefit in the biological control of associated insect and acarine pests. Twenty mite species, belong to 3 suborders representing 11 families and 16 genera were collected (Tables 1-7).

\section{I - Predaceous mites:}

The predaceous mites included 13 species representing 7 families. One family belongs to 
suborder Gamasida and 6 families of the suborder Actinedida

Family Ascidae Voigts \& Oudemans

Two species: Blattisocius keegani Fox and $B$. tarsalis (Berlese) were isolated from the aforementioned stored grains associated with acarid mites and different insects (Table 1). The highest occurrence of this family was noticed in maize (Table 2), and it was frequently found all-over the year in Gharbeya (Table 3).

Blattisocius sp. is of good potential use as predator to control stored grain pests. Barker (1967), cited that immature stages and adults of this predator fed on eggs of numbers of different grain beetles: Tribolium confusum (Jacqueline du Val), $T$. castaneum Herbst, Trygoderma sp. and Oryzaephilus surinamensis Linnaeus. Lindquist (1983), stated that B. tarsalis exserted effective natural control against stored product pyralid moth, especially Anagasta cautella (Walker). In Egypt, (Fawzy 1996), reared Blattisocius keegani Fox on two stored grain pests: Suidasia nesbitti (Hughes) and Grammolichus aegypticus Shereef \& Fawzy. The adult female lived for 25.3 and 26 days and deposited averages of 24.9 and 14.1 eggs when fed on the two aforementioned preys, respectively. The adult female consumed 31 and 25 individuals of $S$. nesbitti and. of G. aegypticus respectively during its life span. Also, (El-Sanady 2005), reared the same predator on the larval stages of Tyrophagus putrescentiae (Schrank) and Rhizoglyphus robini Claparède. The adult consumed 31.1 and 28.5 individuals of the two prementioned preys, respectively, during its life span.

\section{Family Cheyletidae Leach}

Five species were recorded: Cheyletus malaccensis Oudemans, C. malayensis Cunliffe, C. fortis Oudemans, Acaropsellina sollers Rohdendorf and Nodele simplex Wafa \& Soliman. They were found associated with different insect pests and with astigmatid mites on which they feed in different seeds and areas (Tables 1-7).

Cheyletus malaccensis: This species was the most widely distributed predator in this family and the second common mite of all stored Acari. This view is confirmed by (Hoda et al. 1990). It was recorded in the five sources under study. Its highest population was recorded in maize followed by wheat from Kafr El-Sheikh and Gharbeya.

Biological work on this species was undertaken by (Saleh et al. 1986). The immature stages and adult consumed 44 and 23. 7 individuals of the stored product mite: Aleuroglyphus ovatus Troupeau respe-ctively.

Cheyletus malayensis: It was found only in Dakahleya, in all sources under investigation but, in rare number (Table 2). (Hoda et al. 1990) collected it before from rice in Giza, Egypt.

Cheyletus fortis: It was noted in few numbers in wheat, rice and maize from Kafr El-Sheikh only.

Acaropsellina sollers: Few numbers were recorded from bean in Dakahleya. It feeds on acarid mites and their eggs (Summers \& Price 1970).

Nodele simplex: This species was separated from rice in few numbers in Dakahleya.

\section{Family Bdellidae Dugès}

Two species belonging to this family: Spinibdella bifurcate Atyeo and $S$. depressa (Ewing) were recorded.

Spinibdella bifurcate: It was collected from maize, wheat and broad bean seeds in few numbers, from Gharbeya; and was also found in rice from Dakahleya, while Spinibdella depressa collected from broad bean in Gharbeya, (Atyeo 1960) and (Hughes 1961) cited that S. bifurcate prey on arthropod eggs and on other mites.

\section{Family Cunaxidae Thor}

Only Cunaxa capreolus (Berlese) was detected in this family. It was found in rice rough in the three governorates, Gharbeya, Dakahleya and Kafr ElSheikh; in wheat from Menoufeya; and in maize and broad bean from Kafr El-sheikh.

This species was reared by Zaher et al. (1975) on book lice. (Lindquist 1983) stated that members of the family Cunaxidae, are used in biological control.

\section{Family Raphignathidae Kramer}

Incidence of this predatory mite Raphignathus sp. was recorded rarely in maize and wheat, in Menoufeya and Kafr El-Sheikh, associated with other mites and insects. It was noted before by Zaher et al. (1985) and Hoda et al. (1990) in grains in Fayoum and Giza respectively.

\section{Family Stigmaeidae Oudemans}

The predator Apostigmaeus aegyptiacus Soliman \& Gomaa was abundant in rice only in Kafr ElSheikh, associated with acarid mites. Members of this family are regarded as beneficial species. They prey on mites and other associated arthropods (Gonzalez,1965).

\section{Family Pyemotidae Oudemans}

Only two individuals of Pyemotes ventricosus Hughes were separated from maize and flour, in Menoufeya and Dakahleya.

Members of this family are mainly parasitic on insects, sucking their haemolymph. Biological study of this parasite was carried out by Tawfic \& AwadAllah (1970) in Egypt. They recorded that Pyemotes herfsi (Oudemans) limited the population of the pink boll worm, Pectinophora gossypiella Saunders, which passing the winter and the spring in dry cotton boll. as $48 \%$ of the caterpillars were killed. Also, Moser (1975) recorded that P. ventricosus was a 
Table (1): Mites associated with insects in stored grains and seeds in four governorates

Governorate $\&$ stored grain

Insect pests (Order : Family )

Pedators

Mites

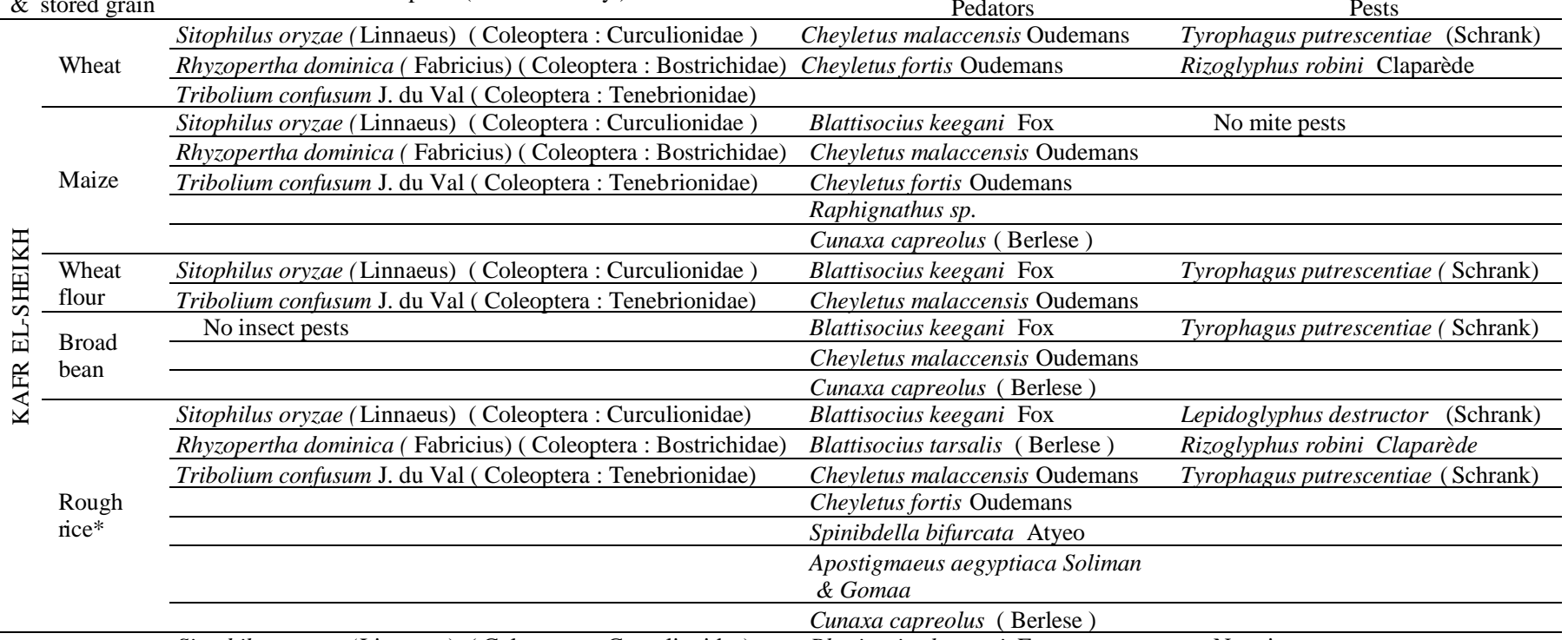

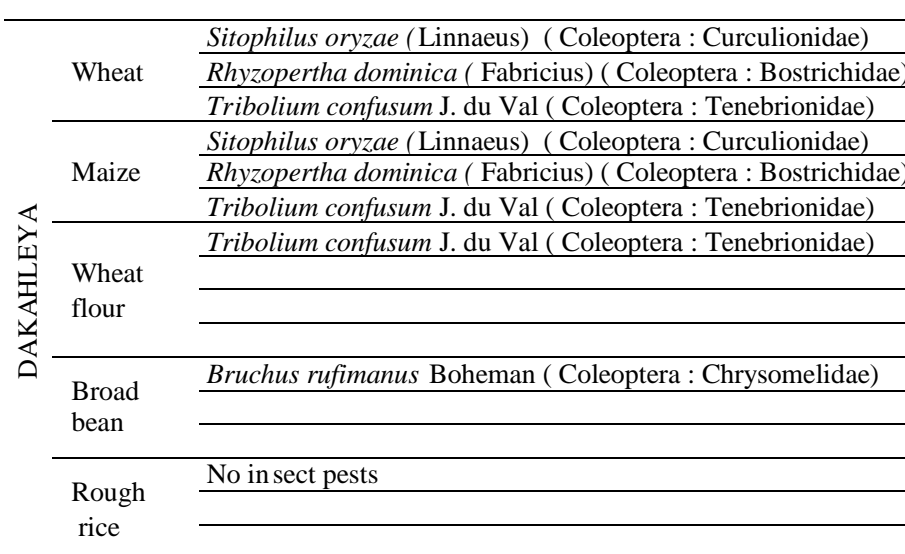

Cunaxa capreolus (Berlese)

Blattisocius keegani Fox

No mite pests

Blattisocius keegani Fox No mite pests

Cheyletus malaccensis Oudemans

Cheyletus malaccensis Oudemans No mite pests

Cheyletus malayensis Cunliffe

Spinibdella bifurcata Atyeo

Pyemotes sp.

Blattisocius keegani Fox

Cheyletus malaccensis Oudemans

Acaropsellina sollers Rhodendorf

Cheyletus malayensis Cunliffe

Spinibdella bifurcata Atyeo

Nodele simplex Wafa \& Soliman

Sitophilus oryzae (Linnaeus) (Coleoptera : Curculionidae) Blattisocius keegani Fox

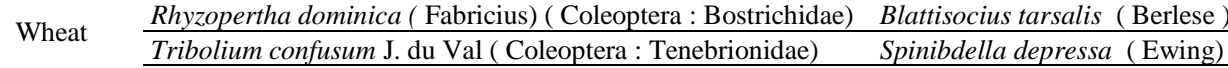

Cheyletus malaccensis Oudemans

Sitophilus oryzae (Linnaeus) (Coleoptera : Curculionidae) Blattisocius keegani Fox

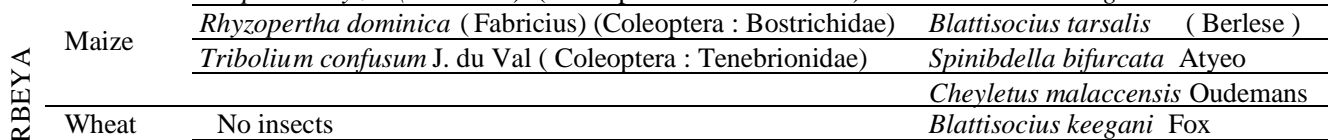

Wheat No insects
flour

J Broad Bruchus rufimanus Boheman (Coleoptera : Chrysomelidae)

bean

Blattisocius keegani Fox

Blattisocius keegani Fox

Spinibdella bifurcata Atyeo

Cheyletus malaccensis Oudemans

Blattisocius keegani Fox

Sitophilus oryzae (Linnaeus) (Coleoptera : Curculionidae)

Rough Rhyzopertha dominica ( Fabricius) (Coleoptera : Bostrichidae)

rice Tribolium confusum J. du Val ( Coleoptera : Tenebrionidae)

Spinibdella bifurcata Atyeo

Cheyletus malaccensis Oudemans

Cunaxa capreolus (Berlese)

Blattisocius keegani Fox

Sitophilus oryzae (Linnaeus) (Coleoptera : Curculionidae)

Wheat

Oryzaephilus suriamensis Linneaus

Blattisocius tarsalis (Berlese)

Cheyletus malaccensis Oudemans

Cunaxa capreolus (Berlese)

Blattisocius keegani Fox

Sitophilus oryzae ( Linnaeus )

Blattisocius tarsalis (Berlese)

Cheyletus malaccensis Oudemans

Cheyletus malayensis Cunliffe

No mite pests

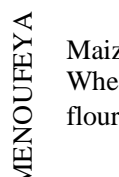

Raphignathus $s p$.

Pyemotes sp.

Broad Tribolium confusum J. du Val (Coleoptera : Tenebrionidae)

bean

Blattisocius tarsalis ( Berlese )

Blattisocius keegani Fox

Rough No insect pests

Cheyletus malaccensis Oudemans

Blattisocius tarsalis (Berlese)

rice

Cheyletus malaccensis Oudemans

Lepidoglyphus destructor (Schrank)

Goheria sp.

Lepidoglyphus destructor (Schrank) Rizoglyphus robini Claparède

Lepidoglyphus destructor (Schrank) Rizoglyphus robini Claparède

Dermatophagoides farini Hughes Lepidoglyphus destructor (Schrank) Rizoglyphus robini Claparède Tyrophagus putrescentiae (Schrank) Dermatophagoides farini Hughes Rizoglyphus robini Claparède Acarus siro Linnaeus

Lepidoglyphus destructor (Schrank)

Rizoglyphus robini Claparède

Acarus siro Linnaeus

Tyrophagus putriscentiae

* Rice grain with husk

Chortoglyphus sp.

Rizoglyphus robini Claparède

Rizoglyphs robini Claprede 
Table (2): Frequency of mites in different stored grains and seeds

\begin{tabular}{|c|c|c|c|c|c|c|}
\hline Suborder, Family\& mite sp. & Status & Maize & Wheat & Wheat flour & Broad bean & Rough rice \\
\hline \multicolumn{7}{|l|}{ I - Suborder : Mesostigmata } \\
\hline \multicolumn{7}{|l|}{ Family Ascidae Viogts\& Oud. } \\
\hline a-Blattisocius keegani Fox & Predator & $* * * *$ & $* * *$ & $* *$ & $*$ & $* * *$ \\
\hline $\mathrm{b}-B$. tarsalis Berlese & Predator & $* * *$ & $* *$ & $*$ & $* *$ & $\mathrm{x}$ \\
\hline \multicolumn{7}{|l|}{ II - Suborder : Prostigmata } \\
\hline \multicolumn{7}{|l|}{ Family Cheyletidae Leach } \\
\hline a- Cheyletus malayensis Cunliffe & Predator & $*$ & $*$ & $*$ & $*$ & $*$ \\
\hline b-C. malaccensis Oudemans & Predator & $* * * *$ & $* * *$ & $*$ & $* *$ & $*$ \\
\hline c- C. fortis Oudemans & Predator & $* *$ & $*$ & $\mathrm{x}$ & $\mathrm{x}$ & $*$ \\
\hline d-Acaropsellina sollers Rohdendorf & Predator & $\mathrm{X}$ & $\mathrm{x}$ & $\mathrm{x}$ & $*$ & $\mathrm{x}$ \\
\hline e- Nodele simplex Wafa\&Soliman & Predator & $\mathrm{x}$ & $\mathrm{x}$ & $\mathrm{x}$ & $\mathrm{x}$ & $*$ \\
\hline \multicolumn{7}{|l|}{ Family Bdellidae } \\
\hline a-Spinibdella depressa (Ewing) & Predator & $\mathrm{X}$ & $*$ & $\mathrm{x}$ & $\mathrm{x}$ & $\mathrm{x}$ \\
\hline b-S.bifurcata Atyeo & Predator & $*$ & $*$ & $*$ & $*$ & $*$ \\
\hline \multicolumn{7}{|l|}{ Family Cunaxidae } \\
\hline Cunaxa capreolus (Berlese) & Predator & $*$ & $*$ & $\mathrm{X}$ & $*$ & $*$ \\
\hline \multicolumn{7}{|l|}{ Family Pyemotidae } \\
\hline Pyemotes sp. & Parasite & $*$ & $\mathrm{x}$ & $*$ & $\mathrm{x}$ & $\mathrm{x}$ \\
\hline \multicolumn{7}{|l|}{ Family Raphignathidae (Kramer) } \\
\hline Raphignathus sp. & Predator & $*$ & $*$ & $\mathrm{x}$ & $\mathrm{x}$ & $\mathrm{x}$ \\
\hline \multicolumn{7}{|l|}{ Family Stigmaeidae Oudemans } \\
\hline Apostigmaeus aegyptiacus Soliman\& Gomaa & Predator & $\mathrm{x}$ & $\mathrm{x}$ & $\mathrm{x}$ & $\mathrm{x}$ & $* * *$ \\
\hline \multicolumn{7}{|l|}{ III- Suborder : Astigmata } \\
\hline \multicolumn{7}{|l|}{ Family Acaridae Ewing \& Nesbitt } \\
\hline a - Acarus siro $\mathrm{L}$ & Pest & $* * * *$ & $*$ & $\mathrm{x}$ & $\mathrm{x}$ & $\mathrm{x}$ \\
\hline b-Tyrophagus putrescentiae (Schrank) & Pest & $*$ & $* * * *$ & $* * * *$ & $* * * *$ & $* * *$ \\
\hline c- Rizoglphus robini Claparède & Pest & $*$ & $* * *$ & $* * * *$ & $*$ & $*$ \\
\hline \multicolumn{7}{|l|}{ Family Glycyphagidae Berlese } \\
\hline a-Lepidoglyphus destructor (Schrank) & Pest & $* *$ & $* * *$ & $*$ & $*$ & $* *$ \\
\hline b-Goheria sp. & Pest & $*$ & $\mathrm{x}$ & $\mathrm{x}$ & $\mathrm{x}$ & $\mathrm{x}$ \\
\hline \multicolumn{7}{|l|}{ Family Pyroglyphidae Cunliffe } \\
\hline Dermatophagoides farinae Hughes & Pest & $\mathrm{X}$ & $\mathrm{x}$ & $\mathrm{x}$ & $*$ & $*$ \\
\hline \multicolumn{7}{|l|}{ Family Chortoglyphidae Berlese } \\
\hline Chortoglyphus sp. & Pest & $\mathrm{x}$ & $\mathrm{x}$ & $*$ & $\mathrm{x}$ & $\mathrm{x}$ \\
\hline
\end{tabular}

*: $1-5$ mites/sample (Rare)

***: 10-25 mites/sample (Abundant)

**: 5-10 mites/sample (Moderate)

$* * * *:<100$ mites/sample (Highly abundant)

$\mathrm{x}$ : Not recorded 
Table (3): Survey of mite species inhabiting maize in different governorates (between March 2003 \& February 2004)

\begin{tabular}{|c|c|c|c|c|c|c|}
\hline & Month & Mite species & Menoufeya & Gharbeya & Dakahleya & Kafr El-Sheikh \\
\hline \multirow{31}{*}{ ஜ̊ } & March & - & $\mathrm{x}$ & $\mathrm{x}$ & $\mathrm{x}$ & $\mathrm{x}$ \\
\hline & \multirow{2}{*}{ April } & Cheyletus malayensis & $*$ & $\mathrm{x}$ & $\mathrm{x}$ & $\mathrm{x}$ \\
\hline & & Blattisocius keegani & $\mathrm{x}$ & $\mathrm{x}$ & $*$ & $\mathrm{x}$ \\
\hline & \multirow{2}{*}{ May } & Cheyletus malaccensis & $\mathrm{x}$ & $\mathrm{x}$ & $*$ & $*$ \\
\hline & & Raphignathus sp. & $\mathrm{x}$ & $\mathrm{x}$ & $\mathrm{x}$ & $*$ \\
\hline & June & Cheylelus fortis & $\mathrm{x}$ & $\mathrm{x}$ & $\mathrm{x}$ & $* *$ \\
\hline & \multirow{2}{*}{ July } & Blattisocius tarsalis & $\mathrm{x}$ & $* * *$ & $\mathrm{x}$ & $\mathrm{x}$ \\
\hline & & Blattisocius keegani & $\mathrm{x}$ & $*$ & $\mathrm{x}$ & $\mathrm{x}$ \\
\hline & \multirow{5}{*}{ August } & Blattisocius keegani & $*$ & $* * * *$ & $*$ & $*$ \\
\hline & & Raphignathus sp. & $*$ & $\mathrm{x}$ & $\mathrm{x}$ & $\mathrm{x}$ \\
\hline & & Cheyletus malaccensis & $\mathrm{x}$ & $* * * *$ & $* *$ & $* * *$ \\
\hline & & Cunaxa capreolus & $\mathrm{x}$ & $\mathrm{x}$ & $\mathrm{x}$ & $*$ \\
\hline & & Acarus siro & $* * * *$ & $\mathrm{x}$ & $\mathrm{x}$ & $\mathrm{x}$ \\
\hline & \multirow{4}{*}{ Sept } & Blattisocius keegani & $*$ & $*$ & $*$ & $\mathrm{x}$ \\
\hline & & Cheyletus malaccensis & $\mathrm{x}$ & $*$ & $* *$ & $\mathrm{x}$ \\
\hline & & Spinibdella bifurcata & $\mathrm{x}$ & $*$ & $\mathrm{x}$ & $\mathrm{x}$ \\
\hline & & Lepidoglyphus destructor & $\mathrm{x}$ & $*$ & $\mathrm{x}$ & $\mathrm{x}$ \\
\hline & \multirow{6}{*}{ Oct } & Blattisocius keegani & $* *$ & $* * * *$ & $*$ & $*$ \\
\hline & & Cheyletus malaccensis & $*$ & $*$ & $\mathrm{x}$ & $*$ \\
\hline & & Pyemotes sp. & $*$ & $\mathrm{x}$ & $\mathrm{x}$ & $\mathrm{x}$ \\
\hline & & Rizoglyphus robini & $*$ & $\mathrm{x}$ & $\mathrm{x}$ & $\mathrm{x}$ \\
\hline & & Lepidoglyphus destructor & $*$ & $\mathrm{x}$ & $\mathrm{x}$ & $\mathrm{x}$ \\
\hline & & Tyrophagus putrescentiae & $*$ & $\mathrm{x}$ & $\mathrm{x}$ & $\mathrm{x}$ \\
\hline & \multirow{5}{*}{ Nov } & Blattisocius keegani & $*$ & $* * *$ & $*$ & $*$ \\
\hline & & Cheyletus malaccensis & $*$ & $*$ & $\mathrm{x}$ & $*$ \\
\hline & & Rizoglyphus robini & $*$ & $\mathrm{x}$ & $\mathrm{x}$ & $\mathrm{x}$ \\
\hline & & Lepidoglyphus destructor & $*$ & $\mathrm{x}$ & $\mathrm{x}$ & $\mathrm{x}$ \\
\hline & & Tyrophagus putrescentiae & $*$ & $\mathrm{x}$ & $\mathrm{x}$ & $\mathrm{x}$ \\
\hline & \multirow{3}{*}{ Dec } & Blattisocius keegani & $*$ & $*$ & $*$ & $*$ \\
\hline & & Rizoglyphus robini & $*$ & $\mathrm{x}$ & $\mathrm{x}$ & $\mathrm{x}$ \\
\hline & & Tyrophagus putrescentiae & $*$ & $*$ & $\mathrm{x}$ & $\mathrm{x}$ \\
\hline \multirow{7}{*}{ ঠ্ণ } & \multirow{4}{*}{ Jan } & Blattisocius keegani & $*$ & $*$ & $*$ & I/I/ \\
\hline & & Cheyletus malaccensis & $\mathrm{x}$ & $*$ & $\mathrm{x}$ & I/II \\
\hline & & Lepidoglyphus destructor & $*$ & $* *$ & $\mathrm{x}$ & $/ / / /$ \\
\hline & & Goheria sp. & $\mathrm{x}$ & $*$ & $\mathrm{x}$ & $/ / / /$ \\
\hline & \multirow{3}{*}{ Feb } & Blattisocius keegani & $*$ & $*$ & $*$ & $/ / / /$ \\
\hline & & Cheyletus malaccensis & $\mathrm{x}$ & $*$ & $\mathrm{x}$ & IIII \\
\hline & & Lepidoglyphus destructor & $*$ & $* *$ & $\mathrm{x}$ & I/II \\
\hline
\end{tabular}

*: 1-5 mites/sample (Rare)

***: 10-25 mites/sample (Abundant)

**: 5-10 mites/sample (Moderate)

****: < 100 mites/sample (Highly abundant)

$\mathrm{x}$ : Not recorded

I/I/: Samples were not available 
Table (4): Survey of mite species inhabiting wheat in different governorates (between March 2003 \& February 2004)

\begin{tabular}{|c|c|c|c|c|c|c|}
\hline & Month & Mite species & Menoufeya & Gharbeya & Dakahleya & Kafr El-Sheikh \\
\hline \multirow{31}{*}{ 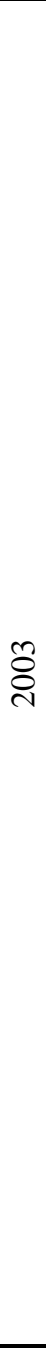 } & March & & $\mathrm{x}$ & $\mathrm{x}$ & $\mathrm{x}$ & $\mathrm{x}$ \\
\hline & \multirow{3}{*}{ April } & Cheyletus malaccensis & $\mathrm{x}$ & $*$ & $\mathrm{x}$ & $\mathrm{x}$ \\
\hline & & Cunaxa capreolus & $*$ & $\mathrm{x}$ & $\mathrm{x}$ & $\mathrm{x}$ \\
\hline & & Spinibdella depressa & $\mathrm{x}$ & $*$ & $\mathrm{x}$ & $\mathrm{x}$ \\
\hline & \multirow{3}{*}{ May } & Cheyletus malayensis & $\mathrm{x}$ & $\mathrm{x}$ & $*$ & $\mathrm{x}$ \\
\hline & & C. malaccensis & $\mathrm{x}$ & $\mathrm{x}$ & $\mathrm{x}$ & $*$ \\
\hline & & Raphignathus & $\mathrm{x}$ & $\mathrm{x}$ & $\mathrm{x}$ & $*$ \\
\hline & \multirow{3}{*}{ June } & Cheyletus fortis & $\mathrm{x}$ & $\mathrm{x}$ & $x$ & $*$ \\
\hline & & Blattisocius keegani & $\mathrm{x}$ & $*$ & $\mathrm{x}$ & $\mathrm{x}$ \\
\hline & & B. tarsalis & $\mathrm{x}$ & $*$ & $\mathrm{x}$ & $\mathrm{x}$ \\
\hline & \multirow{4}{*}{ July } & B. tarsalis & $* *$ & $\mathrm{x}$ & $\mathrm{x}$ & $\mathrm{x}$ \\
\hline & & B.keegani & $\mathrm{x}$ & $*$ & $\mathrm{x}$ & $\mathrm{x}$ \\
\hline & & Cheyletus malaccensis & $\mathrm{x}$ & $* * *$ & $\mathrm{x}$ & $*$ \\
\hline & & Spinibdella bifurcata & $\mathrm{x}$ & $*$ & $x$ & $\mathrm{x}$ \\
\hline & \multirow{3}{*}{ August } & Blattisocius keegani & $\mathrm{x}$ & $*$ & $*$ & $\mathrm{x}$ \\
\hline & & Cheyletus malaccensis & $*$ & $* *$ & $* *$ & $* *$ \\
\hline & & Acarus siro & $*$ & $\mathrm{x}$ & $\mathrm{x}$ & $\mathrm{x}$ \\
\hline & \multirow{4}{*}{ Sept } & Blattisocius keegani & $*$ & $\mathrm{x}$ & $\mathrm{x}$ & $\mathrm{x}$ \\
\hline & & B. tarsalis & $*$ & $* * *$ & $\mathrm{x}$ & $\mathrm{x}$ \\
\hline & & Cheyletus malaccensis & $*$ & $* *$ & $\mathrm{x}$ & $* * *$ \\
\hline & & Lepidoglyphus destructor & $\mathrm{x}$ & $* *$ & $\mathrm{x}$ & $\mathrm{x}$ \\
\hline & \multirow{2}{*}{ Oct } & Cheyletus malaccensis & $\mathrm{x}$ & $*$ & $\mathrm{x}$ & $\mathrm{x}$ \\
\hline & & Rhizoglyphus robini & $*$ & & $\mathrm{x}$ & $\mathrm{x}$ \\
\hline & \multirow{4}{*}{ Nov } & Blattisocius keegani & $\mathrm{x}$ & $*$ & $\mathrm{x}$ & $\mathrm{x}$ \\
\hline & & Cheyletus malaccensis & $\mathrm{x}$ & $*$ & $*$ & $\mathrm{x}$ \\
\hline & & Rhizoglyphus robini & $*$ & $\mathrm{x}$ & $\mathrm{x}$ & $* *$ \\
\hline & & Lepidoglyphus destructor & $\mathrm{x}$ & $*$ & $\mathrm{x}$ & $\mathrm{x}$ \\
\hline & \multirow{4}{*}{ Dec } & Blattisocius keegani & $\mathrm{x}$ & $*$ & $*$ & $\mathrm{x}$ \\
\hline & & B. tarsalis & $\mathrm{x}$ & $*$ & $\mathrm{x}$ & $\mathrm{x}$ \\
\hline & & Cheyletus malaccensis & $\mathrm{x}$ & $\mathrm{x}$ & $\mathrm{x}$ & $* *$ \\
\hline & & Rhizoglyphus robini & $\mathrm{x}$ & $\mathrm{x}$ & $\mathrm{x}$ & $* *$ \\
\hline \multirow{13}{*}{ 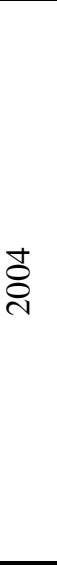 } & \multirow{7}{*}{ Jan } & Blattisocius keegani & $\mathrm{x}$ & $*$ & $*$ & $\mathrm{x}$ \\
\hline & & B. tarsalis & $\mathrm{x}$ & $*$ & $\mathrm{x}$ & $\mathrm{x}$ \\
\hline & & Cheyletus malaccensis & $*$ & $\mathrm{x}$ & $*$ & $* * *$ \\
\hline & & Lepidoglyphus destructor & $\mathrm{x}$ & $* * *$ & $\mathrm{x}$ & $\mathrm{x}$ \\
\hline & & Tyrophagus putrescentiae & $\mathrm{x}$ & $\mathrm{x}$ & $\mathrm{x}$ & $* * * *$ \\
\hline & & Rhizoglyphus robini & $*$ & $\mathrm{x}$ & $\mathrm{x}$ & $* * * *$ \\
\hline & & Acarus siro & $\mathrm{x}$ & $*$ & $\mathrm{x}$ & $\mathrm{x}$ \\
\hline & \multirow{6}{*}{ Feb } & Blattisocius keegani & $\mathrm{x}$ & $*$ & $*$ & $\mathrm{x}$ \\
\hline & & Cheyletus malaccensis & $*$ & $\mathrm{x}$ & $*$ & $* *$ \\
\hline & & Lepidoglyphus destructor & $\mathrm{x}$ & $* *$ & $x$ & $\mathrm{x}$ \\
\hline & & Tyrophagus putrescentiae & $\mathrm{x}$ & $\mathrm{x}$ & $\mathrm{x}$ & $* * *$ \\
\hline & & Rhizoglyphus robini & $*$ & $\mathrm{x}$ & $\mathrm{x}$ & $* * *$ \\
\hline & & Acarus siro & $\mathrm{x}$ & $*$ & $\mathrm{x}$ & $\mathrm{x}$ \\
\hline
\end{tabular}

*: 1 - 5 mites / sample (Rare)

**: 5 - 10 mites / sample (Moderate)

***: 10 - 25 mites / sample (Abundant)

****: < 100 mites / sample (Highly abundant)

$\mathrm{x}$ : Not recorded 
Table (5): Survey of mite species inhabiting wheat flour in different governorates (between March 2003 \& February 2004)

\begin{tabular}{|c|c|c|c|c|c|c|}
\hline & Month & Mite species & Menoufeya & Gharbeya & Dakahleya & Kafr El-Sheikh \\
\hline \multirow{23}{*}{ 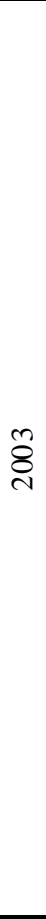 } & March & & $\mathrm{x}$ & $\mathrm{x}$ & $\mathrm{x}$ & //I/ \\
\hline & April & & $\mathrm{x}$ & $\mathrm{x}$ & $\mathrm{x}$ & $\mathrm{x}$ \\
\hline & May & & $\mathrm{x}$ & $\mathrm{X}$ & $\mathrm{x}$ & $\mathrm{x}$ \\
\hline & June & & $\mathrm{x}$ & $\mathrm{x}$ & $\mathrm{x}$ & $\mathrm{x}$ \\
\hline & \multirow{4}{*}{ July } & Blattisocius tarsalis & $*$ & $\mathrm{X}$ & $\mathrm{X}$ & $\mathrm{X}$ \\
\hline & & Cheyletus malaccensis & $*$ & $\mathrm{X}$ & $\mathrm{X}$ & $\mathrm{X}$ \\
\hline & & C. malayensis & $\mathrm{x}$ & $\mathrm{X}$ & $*$ & $\mathrm{X}$ \\
\hline & & Spinibdella bifurcata & $\mathrm{x}$ & $\mathrm{x}$ & $*$ & $\mathrm{x}$ \\
\hline & August & Cheyletus malaccensis & $*$ & $\mathrm{x}$ & $\mathrm{x}$ & $*$ \\
\hline & \multirow{3}{*}{ Sept } & Blattisocius keegani & $\mathrm{x}$ & $*$ & $\mathrm{x}$ & $\mathrm{x}$ \\
\hline & & Pyemotes sp. & $\mathrm{x}$ & $\mathrm{x}$ & $\mathrm{x}$ & $\mathrm{x}$ \\
\hline & & Lepidoglyphus destructor & $\mathrm{x}$ & $\mathrm{X}$ & $\mathrm{x}$ & $\mathrm{x}$ \\
\hline & \multirow{4}{*}{ Oct } & Blattisocius keegani & $*$ & $\mathrm{x}$ & $\mathrm{X}$ & $*$ \\
\hline & & Cheyletus malaccensis & $\mathrm{X}$ & $\mathrm{x}$ & $*$ & $\mathrm{X}$ \\
\hline & & Chortoglyphus sp. & $*$ & $\mathrm{x}$ & $\mathrm{X}$ & $\mathrm{X}$ \\
\hline & & Rhizoglyphus robini & $\mathrm{X}$ & $* * * *$ & $\mathrm{x}$ & $\mathrm{x}$ \\
\hline & \multirow{3}{*}{ Nov } & Blattisocius keegani & $*$ & $*$ & $\mathrm{x}$ & $*$ \\
\hline & & Chortoglyphus sp. & $*$ & $\mathrm{x}$ & $\mathrm{x}$ & $\mathrm{x}$ \\
\hline & & Rhizoglyphus robini & $\mathrm{x}$ & $* * *$ & $\mathrm{X}$ & $\mathrm{x}$ \\
\hline & \multirow{4}{*}{ Dec } & Blattisocius keegani & $*$ & $*$ & $\mathrm{X}$ & $\mathrm{X}$ \\
\hline & & Rhizoglyphus robini & $\mathrm{x}$ & $* *$ & $\mathrm{x}$ & $\mathrm{X}$ \\
\hline & & Lepidoglyphus destructor & $\mathrm{x}$ & $*$ & $\mathrm{x}$ & $\mathrm{x}$ \\
\hline & & Tyrophagus putrescentiae & $\mathrm{X}$ & $*$ & $\mathrm{X}$ & $\mathrm{X}$ \\
\hline \multirow{3}{*}{$\underset{\sim}{\stackrel{\Xi}{8}}$} & \multirow{2}{*}{ Jan } & Lepidoglyphus destructor & $\mathrm{x}$ & $*$ & $\mathrm{x}$ & $\mathrm{X}$ \\
\hline & & Tyrophagus putrescentiae & $\mathrm{x}$ & $\mathrm{x}$ & $\mathrm{x}$ & $* * * *$ \\
\hline & Feb & Tyrophagus putrescentiae & $\mathrm{x}$ & $*$ & $\mathrm{x}$ & $* * *$ \\
\hline
\end{tabular}

Table (6): Survey of mite species inhabiting broad bean seeds in different governorates (between March 2003 \& February 2004)

\begin{tabular}{|c|c|c|c|c|c|c|}
\hline & Month & Mite species & Menoufeya & Gharbeya & Dakahleya & Kafr El-Sheikh \\
\hline \multirow{24}{*}{ లి } & March & & $\mathrm{X}$ & $\mathrm{X}$ & $\mathrm{X}$ & $\mathrm{X}$ \\
\hline & April & Acaropsellina sollers & $\mathrm{x}$ & $\mathrm{x}$ & $*$ & $\mathrm{x}$ \\
\hline & May & Acaropsellina sollers & $\mathrm{x}$ & $\mathrm{x}$ & $*$ & $\mathrm{x}$ \\
\hline & June & Spinibdella bifurcata & $\mathrm{x}$ & $*$ & $\mathrm{x}$ & $\mathrm{x}$ \\
\hline & \multirow{5}{*}{ July } & Blattisocius tarsalis & $* *$ & $\mathrm{x}$ & $\mathrm{x}$ & $\mathrm{x}$ \\
\hline & & Cheyletus malaccensis & $*$ & $\mathrm{x}$ & $\mathrm{x}$ & $\mathrm{x}$ \\
\hline & & C. malayensis & $\mathrm{x}$ & $\mathrm{x}$ & $*$ & $\mathrm{x}$ \\
\hline & & Cunaxa capreolus & $\mathrm{x}$ & $\mathrm{x}$ & $\mathrm{x}$ & $*$ \\
\hline & & Spinibdella bifurcata & $\mathrm{x}$ & $*$ & $\mathrm{x}$ & $\mathrm{x}$ \\
\hline & \multirow{2}{*}{ August } & Blattisocius keegani & $\mathrm{x}$ & $\mathrm{x}$ & $\mathrm{x}$ & $*$ \\
\hline & & Cheyletus malaccensis & $* * *$ & $\mathrm{x}$ & $* *$ & $* *$ \\
\hline & \multirow{4}{*}{ Sept } & Blattisocius keegani & $\mathrm{x}$ & $*$ & $\mathrm{x}$ & $\mathrm{x}$ \\
\hline & & Cheyletus malaccensis & $*$ & $\mathrm{x}$ & $\mathrm{x}$ & $\mathrm{x}$ \\
\hline & & Dermatophagoides farinae & $\mathrm{x}$ & $*$ & $\mathrm{x}$ & $\mathrm{x}$ \\
\hline & & Lepidoglyphus destructor & $\mathrm{x}$ & $*$ & $\mathrm{x}$ & $\mathrm{x}$ \\
\hline & \multirow{3}{*}{ Oct } & Blattisocius keegani & $\mathrm{x}$ & $\mathrm{x}$ & $*$ & $*$ \\
\hline & & Cheyletus malaccensis & $*$ & $*$ & $\mathrm{x}$ & $\mathrm{x}$ \\
\hline & & Rhizoglyphus robini & $*$ & $*$ & $\mathrm{x}$ & $\mathrm{x}$ \\
\hline & \multirow{2}{*}{ Nov } & Blattisocius keegani & $\mathrm{x}$ & $\mathrm{x}$ & $\mathrm{x}$ & $*$ \\
\hline & & Rhizoglyphus robini & $\mathrm{x}$ & $*$ & $\mathrm{x}$ & $\mathrm{x}$ \\
\hline & \multirow{4}{*}{ Dec } & Blattispcius keegani & $*$ & $*$ & $\mathrm{x}$ & $*$ \\
\hline & & Rhizoglyphus robini & $*$ & $*$ & $\mathrm{x}$ & $\mathrm{X}$ \\
\hline & & Tyrophagus putrescentiae & $\mathrm{x}$ & $\mathrm{x}$ & $\mathrm{x}$ & $* * * *$ \\
\hline & & Lepidoglyphus destructor & $\mathrm{x}$ & $\mathrm{x}$ & $\mathrm{x}$ & $\mathrm{x}$ \\
\hline \multirow{3}{*}{$\begin{array}{l}\text { ঠ্ } \\
\text { ¿ }\end{array}$} & \multirow{3}{*}{ Jan } & Blattisocius keegani & $\mathrm{x}$ & $*$ & $\mathrm{x}$ & $\mathrm{x}$ \\
\hline & & Cheyletus malaccensis & $\mathrm{x}$ & $\mathrm{x}$ & $\mathrm{x}$ & $*$ \\
\hline & & Lepidoglyphus destructor & $\mathrm{x}$ & $* * *$ & $\mathrm{x}$ & $\mathrm{x}$ \\
\hline
\end{tabular}


Table (7): Survey of mite species inhabiting rough rice in different governorates (between March 2003 \& February 2004)

\begin{tabular}{|c|c|c|c|c|c|}
\hline & Month & Mite species & Gharbeya & Dakahleya & Kafr El-Sheikh \\
\hline \multirow{29}{*}{ శ్ } & March & & $\mathrm{x}$ & $\mathrm{x}$ & $\mathrm{x}$ \\
\hline & April & & $\mathrm{x}$ & $\mathrm{x}$ & $\mathrm{x}$ \\
\hline & May & & $\mathrm{x}$ & $\mathrm{x}$ & $\mathrm{x}$ \\
\hline & \multirow{6}{*}{ June } & Blattisocius keegani & $*$ & $\mathrm{x}$ & $\mathrm{x}$ \\
\hline & & Nodele simplex & $\mathrm{x}$ & $*$ & $\mathrm{x}$ \\
\hline & & Cheyletus fortis & $\mathrm{x}$ & $\mathrm{x}$ & $*$ \\
\hline & & Cunaxa capreolus & $\mathrm{x}$ & $\mathrm{x}$ & $*$ \\
\hline & & Spinibdella bifurcata & $\mathrm{x}$ & $*$ & $\mathrm{x}$ \\
\hline & & Tyrophagus putrescentiae & $\mathrm{x}$ & $\mathrm{x}$ & $\mathrm{x}$ \\
\hline & \multirow{5}{*}{ July } & Blattisocius keegani & $*$ & $\mathrm{x}$ & $\mathrm{x}$ \\
\hline & & Cheyletus malayensis & $\mathrm{x}$ & $*$ & $\mathrm{x}$ \\
\hline & & Spinibdella bifurcata & $\mathrm{x}$ & $*$ & $\mathrm{x}$ \\
\hline & & Cunaxa capreolus & $\mathrm{x}$ & $\mathrm{x}$ & $*$ \\
\hline & & Tyrophagus putrescentiae & $*$ & $\mathrm{x}$ & $\mathrm{x}$ \\
\hline & \multirow{2}{*}{ Aug. } & Cheyletus malayensis & $\mathrm{x}$ & $*$ & $\mathrm{x}$ \\
\hline & & Spinibdella bifurcata & $*$ & $*$ & $\mathrm{x}$ \\
\hline & \multirow{4}{*}{ Sept. } & Blattisocius keegani & $* * *$ & $\mathrm{x}$ & $\mathrm{x}$ \\
\hline & & Cheyletus malaccensis & $*$ & $\mathrm{x}$ & $*$ \\
\hline & & Spinibdella bifurcata & $*$ & $\mathrm{x}$ & $\mathrm{x}$ \\
\hline & & Lepidoglyphus destructor & $\mathrm{x}$ & $\mathrm{x}$ & $*$ \\
\hline & \multirow{4}{*}{ Oct. } & Blattisocius keegani & $* *$ & IIII & $*$ \\
\hline & & Cheyletus malaccensis & $\mathrm{x}$ & IIII & $*$ \\
\hline & & Cunaxa capreolus & $*$ & $\| / I /$ & $\mathrm{x}$ \\
\hline & & Rhizoglyphus robini & $*$ & $/ I / I$ & $*$ \\
\hline & \multirow{5}{*}{ Nov. } & Blattisocius keegani & $*$ & $/ / I /$ & $*$ \\
\hline & & Cheyletus malaccensis & $\mathrm{x}$ & IIII & $*$ \\
\hline & & Rhizoglyphus robini & $*$ & IIII & $*$ \\
\hline & & Lepidoglyphus destructor & $*$ & $I / I /$ & $* *$ \\
\hline & & Tyrophagus putrescentiae & $\mathrm{x}$ & IIII & $* * *$ \\
\hline \multirow{8}{*}{ ষ্ণ } & \multirow{8}{*}{ Jan. } & Blattisocius keegani & $*$ & IIII & $*$ \\
\hline & & Blattisocius tarsalis & $\mathrm{x}$ & IIII & $*$ \\
\hline & & Cheyletus malaccensis & $\mathrm{x}$ & $1 / I /$ & $*$ \\
\hline & & Apostigmaeus sp. & $\mathrm{x}$ & IIII & $* *$ \\
\hline & & Lepidoglyphus destructor & $*$ & IIII & $* *$ \\
\hline & & Rhizoglyphus robini & $*$ & $/ / I /$ & $*$ \\
\hline & & Dermatophagoides farinae & $*$ & IIII & $* * *$ \\
\hline & & Tyrophagus putrescentiae & $\mathrm{x}$ & IIII & $* * *$ \\
\hline
\end{tabular}

*: 1 - 5 mites / sample (Rare)

**: 5 - 10 mites / sample (Moderate)

$* * *: 10$ - 25 mites / sample (Abundant)

****: < 100 mites / sample (Highly abundant)

//I/: Samples were not available

$\mathrm{x}$ : Not recorded 
common predator of larval and pupal moths in grain storages attacking granary.

\section{II - Grains feeders and fungivorous mites}

Four families belonging to suborder Acaridida were recorded namely: Acaridae, Glycyphagidae, Pyroglyphidae and Chortogly-phidae.

Members of these families may cause direct injury by feeding and loosing weight of stored grains, and by contamination with old skins, excrement and dead bodies; also feed on fungi. Infested materials became unsuitable for human consumption and may cause digestive troubles when eaten and dermatitis when handled (Sinha, 1964).

\section{Family Acaridae Ewing \& Nesbitt}

Three acarid species of this family namely, Tyrophagus putrescentiae (Schrank), Rhizoglyphus robini Claparède and Acarus siro Linnaeus were noted.

T. putrescentiae and $R$. robini were noted in great numbers in the five sources under study. Developmental stages of these species were found in wheat, flour and bread bean, in Gharbeya and Kafr El-Sheikh (Tables 4-6). This finding was indicated previously by Wafa et al., 1966; Krantz 1978; Fawzy 1996 and El-Sanady 2005). Hughes 1961 reported that $T$. putrescentiae completed its life cycle, in 2 to 3 weeks, on wheat germ as food. In 1968, Sinha and Mills noted that $\boldsymbol{A}$. siro fed on Penicillium sp. and distributed the spores throughout the substrate.

\section{Family Glycyphagidae Berlese}

Two glycyphagid species were recorded, Lepidoglyphus destructor(Schrank) and Gohieria Oudemans.

Lepidoglyphus destructor was frequently found in the five aforementioned stored grains. Similar result was obtained by (Fawzy, 1996). It could cause allergy when handled.

Gohieria sp.: It was collected from maize in Gharbeya. It is widely distributed in stored grains in many parts of the world (Hughes, 1976).

\section{Family Pyroglyphidae Cunliffe}

Only one species was recorded, Dermatophagoides farinae Hughes. It was isolated from rice and bean from Gharbeya and Kafr El-Sheikh Governorates (Table 7). It was found in flour by (Attiah 1969) and in corn and flour by (Zaher 1986).

\section{Family Chortoglyphidae Berlese}

Only, the genus Chortoglyphus Berlese was collected from flour in Menoufeya. Hughes, (1976), separated this species from mills and granaries. Also, (Attiah, 1969), isolated it from rice.

However the stored grains harbured predatory and parasitic mites associated with different pest species of mites and insects. Other group fed on grains, seeds and other stored products. Contamination with their dead bodies and excreta as well as other microorganisms make it unsuitable for human use causing digestive troubles and dermatitis. Other mites prefer to feed on fungi.

Research on the potential of predatory and parasitic mites in controlling destructive insects and mites in stored grains should be augmented. The effect of naturally occurring and utilizing predatory mites associated with stored grain insects require further study.

\section{ACKNOWLEDGEMENT}

The authoresses thank all members of the Regional Council for Research and Agriculture Extension ARC, Ministry of Agriculture for financing this project.

\section{REFERENCES}

Attiah, H. H., 1969. Tyroglyphoid mites associated with stored food in United Arabic Republic UAR. Min. Agric. Plant protection Department Tech. Bull. 10; 1-51.

Atyeo, W. T., 1960. A revision of the mite Family Bdellidae in North \& Central America (Acarina: Prostigmata). The University of Science Bulletin ,40: 345-499.

Barker, P. S., 1967. Bionomic of Blattisocius keegani Fox (Acarina: Ascidae), a predator on eggs of pests of stored grain. Can. J.Zool. 45(1): 093-9.

El-Sanady, M. A., 2005. Studies on some stored product mites and their predators. Ph.D. Thesis, 193 pp. Fac. Sc. (girls) Al-Azhar Univ. Egypt.

Fawzy, M. M. H., 1996. Biological studies on house dust mites in Egypt. Ph. D. Thesis, 237pp. Fac. Agric. Cairo Univ.

Gonzalez, P. H., 1965. A taxonomic study of the genera Mediolata, Zetzellia and Agistemus (Acari: Stigmaeidae). Univ. Calif. Publ. Ent. (41): 1- 64.

Halawa, Z.A., 2003. Mites associated with stored food stuffs in Alexandria Governorate. Bull. Ent. Soc. Egypt. 80: 47-58.

Hoda, F.M.; El-Naggar, M.E.; Taha, H. A; and EL-Beheiry, M. M., 1990. Prostigmati mites associated with stored products. Agric. Res. Rev. 68: 77-85.

Hughes, A. M., 1961. The mites of stored food. Tech. Bull. Minist. Agric. Fish. Food, Bull. 9, 287pp. Hughes, A. M. 1976. The mites of stored food and houses Tech. Bull. Minist. Agric. Fish. Food, Bull. 9, 400 pp.

Krantz, G. W., 1978. A manual of Acarology. 
Oregon State Univ. Book Stores Carvallis, Oregon USA.

Lindquist, E. E., 1983. Some thoughts on the potential for use of mites in biological control including a modified concept of "parasitoids", pp.12-20. In M. A.

Hoy., G. h. Cuningham\& h. Knutson [eds] Biological control of pests by mites. Div. Agric. Nat. Res. Spec. Publ. 3304. University of California Berkeley USA.

Moser, J. C., 1975. Biosystematics of the straw itch mite with special reference to nomenclature and dermatology. Tans. Roy. Ent. Soc. London 127 (2): 185-191. ( Pyemotidae).

Saleh, S. M.; El-Helaly, A., and El-Gayar, F. H., 1986. Life history of the predatory mites Cheyletus malaccensis Oudemans. Acarologia, 27 (1) $37-40$.

Sinha, R. N., 1964. Ecological relationships of stored product mites and seed borne fungi, Proc. $1^{\text {st }}$ Int. Congr. Acarology, 1963. Acarologia, 6: 372- 389.

Sinha, R. N. and Mills, J. T. 1968. Feeding and reproduction of the grain mite and the mushroom mite on some species of Penicilium. J. econ. Ent.61 (6): 1548-1552.

Summers, F. M. and Price. D. W., 1970. Review of the mite family Cheyletidae. University of California press Berkeley and Los Angeles,
$153 \mathrm{pp}$.

Taha, H. A., 1985. Morphological and biological studies on some mites associated with stored products. Ph. D. Thesis, 159 pp., Fac. Agric. AlAzhar Univ. Egypt.

Tawfic, M. F. and Awad-Allah, K. T., 1970. The biology of Pyemotes Herfsi Oudemans and its efficiency in the control of the resting larvae of the pink bollworm, Pectinophora gossypiella Saunders in UAR., Bull. Ent. Soc., Egypt. 54:4 9-71.

Wafa, A. K.; Zaher, M. A.; El-Kifl, A. M. and Hegazy M. A., 1966. Survey on stored grain and seed mites (Acarina). Bull. Ent. Soc., Egypt 50: 225-232.

Zaher, M. A., 1986. Survey and ecological studies on phytophagous, Predaceous and soil mites in Egypt. II - A predaceous, non predaceous Mites (Nile Valley \& Delta), PL 480 Progr. \& USA 567 pp.

Zaher, M. A; Mohamed, M. I. And Abdel-Halim, S. M., 1985. Incidence of mites associated with stored seeds and food products in Upper Egypt. Experimental and Applied Acarology (2): 19-24.

Zaher, M. A., Soliman, Z. R. and El-Bishlawy, S. M., 1975. Feeding habits of the predaceous mite Cunaxa capreolus Berlese (Acarina: Cunaxidae). Entomophaga, 25 (2): 209-212. 